\title{
A NEW PORTABLE IRRIGATOR.
}

We have received from Mr. Morton Whitby, Chief Assistant, Genito-Urinary Department, West London Hospital, W. the following note of a new portable irrigator for washing out the large intestine, uterus, or bladder. The apparatus has been made by the Surgical Manufacturing Co. Ltd. 83-85 Mortimer Street. W. to Mr. Whitby's own design. In view of the more extensive use of lavage in recent years we feel that this useful apparatus will be of practical interest to our readers. Mr. Whitby has sent us the following short account.

Intestinal Lavage has for the past few years been

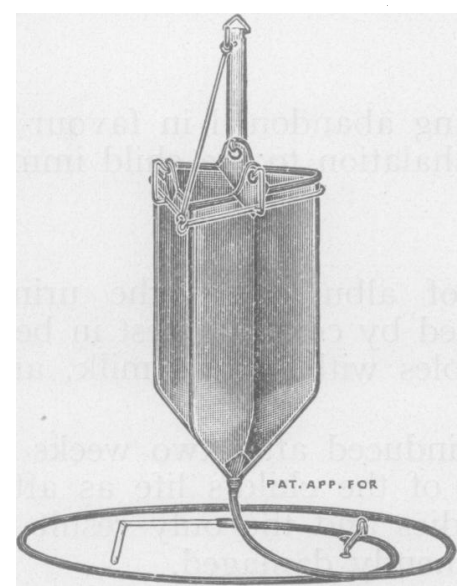
recognised by Urologists, General Surgeons, and Physicians, as the secret of success to all their investigations and treatments.

As a preliminary to X-Ray examination of the kidney, it is undoubtedly the only certain means of excluding gas from the radiograms; a matter of considerable importance in pyelography, etc.

In the treatment of diseases of the urinary system, it is of great value in pyelitis, cystitis and expulsion of small urinary calculii, owing to the profuse diuresis induced.

Preparatory to any operation, it is the surest means of eliminating intestinal toxæmia which is often the cause of disastrous results.

The physician has a long list of diseases for which he uses it as part of the treatment.

Its future in the field of preventive surgery or medicine cannot be too greatly emphasized.

\section{Apparatus and Method of Use.}

The apparatus can be used upon any lavatory seat with a ring cushion. If required for a bed patient, a special rubber bed-pan can be used. In using the apparatus the tube should first be lubricated with vaseline and passed gently into the rectum. The patient sits upon the cushion, with one arm of the tube in the rectum and the other between the buttock and cushion. Thus a firm pressure is exerted to prevent the tube from being pushed out. When the bag is filled with fluid at a body temperature and is suspended 2 or 3 feet above the pelvis, the fluid is allowed to flow slowly into the rectum by means of the regulating clip. The desire to evacuate should be produced by the flow of one pint of fluid in the first instance and a half pint thereafter. As soon as the slightest discomfiture is felt the flow should be stopped, so as to prevent over-distention. This is the only danger. It will generally be found that the bowel will evacuate about ten times to the gallon of fluid, equivalent to about ten days evacuations, in the case of the majority of present day people. The irrigation can be carried out by the patient with perfect safety. The apparatus can be used for intraüterine wash-outs, done by the surgeon, for vaginal or bladder wash-outs with appropriate nozzles by the patient. 